\title{
Vortex shells in mesoscopic superconducting disks
}

\author{
B. J. Baelus, ${ }^{*}$ L. R. E. Cabral ${ }^{\dagger}$ and F. M. Peeters ${ }^{\ddagger}$ \\ Departement Natuurkunde, Universiteit Antwerpen (Campus Drie Eiken), Universiteitsplein 1, B-2610 Antwerpen, Belgium
}

(Received 29 August 2003; published 20 February 2004)

\begin{abstract}
The distribution of vortices over different vortex shells in mesoscopic superconducting disks with sufficiently large sizes is investigated within the framework of the nonlinear Ginzburg-Landau theory. Keeping the total vorticity fixed, different vortex configurations can be found in which the vortices are distributed differently over the vortex shells. An overview is given of the different possible vortex configurations and the free energies of these states are compared. In general, the difference in the free energy between the possible vortex configurations with the same vorticity is much smaller than the difference in the free energy between vortex states with different vorticity. The transitions between different vortex states with the same and different vorticity are investigated. Contrary to small disks, the change in vorticity can be larger than one with increasing and decreasing magnetic field. Also a combination of a giant vortex state with a multivortex state can nucleate into a stable vortex state. The influence of the sample thickness is briefly studied. Our results are compared with those obtained from the London approximation which clearly shows the limited applicability of the latter.
\end{abstract}

DOI: 10.1103/PhysRevB.69.064506

PACS number(s): 74.20.De, 74.25.Dw, 74.25.Ha

\section{INTRODUCTION}

Vortices can be viewed as a specific type of quasiparticles interacting magnetically and through the encircling superconducting screening currents with each other. This makes confined vortex structures somewhat similar to other systems such as electrons in artificial atoms that exhibit complex selforganized patterns for a small number of electrons. ${ }^{1,2}$ Similarly to Hund's rule, it is expected that vortices will obey specific rules for shell filling and exhibit magic numbers. But, on the other hand, the present situation is expected to be complicated by the fact that vortices are not pointlike objects. They can overlap and, contrary to electrons in artificial atoms, even join together creating a giant vortex state.

The aim of the present paper is to determine the vortex positions in mesoscopic disks with sufficiently large radius, such that different rings of vortices can appear. Until now, only limiting cases were studied thoroughly: (1) disks with small radii in which at most a single ring of vortices can be present (see, for example, Refs. 3-9), and (2) infinitely extended superconducting films where the triangular Abrikosov vortex lattice is energetically favorable (see, for example, Refs. 10-12). Similar as for classical charged particles (see, e.g., Ref. 2), one expects that in the intermediate region first more rings (or shells) of vortices will appear with increasing radius, and, for sufficiently large radius, a triangular lattice is formed in the center of the disk, i.e., far away from the edge of the superconductor.

The present paper will be limited to the situation where several shells of vortices will nucleate, but the triangular lattice is not yet formed in the center. We investigate how the vortices can be distributed in different ways over the different shells and which vortex configuration is energetically most favorable. We also investigate the transition between different vortex states.

Previously, this intermediate region was studied using more restrictive approximations. Buzdin and Brison ${ }^{13}$ studied vortex structures in superconducting disks using the image method, where vortices are considered to be pointlike particles, i.e., the London approximation. They calculated the magnetization and the optimum vortex configuration as well as the entrance field. Palacios calculated the vortex configurations in superconducting mesoscopic disks with radius equal to $R=8.0 \xi$, where two vortex shells can become stable. ${ }^{14} \mathrm{He}$ expanded the order parameter in normalized nodeless functions that diagonalize the kinetic-energy operator and limited the number of contributing terms to 3 or 4 . $\mathrm{He}$ also neglected the demagnetization effects by assuming the magnetic field everywhere equal to some effective field. In a previous paper we also briefly studied the vortex configurations in superconducting disks with $d \ll \lambda, \xi$ and $R$ $=6.0 \xi$ and expanded the order parameter over all eigenfunctions with energy $\epsilon_{i}<\epsilon_{*}$, where the cutting energy $\epsilon_{*}$ was chosen such that increasing it does not influence the results. ${ }^{15}$ This work is more general than Ref. 14 because also states with radial nodes were included. It was assumed that the magnetic field equals the applied field everywhere and furthermore the main topic of this paper were the saddle-point transitions between different vortex states in disks and rings. Geim et al. ${ }^{16}$ studied experimentally and theoretically the magnetization of different vortex configurations in superconducting disks. They found clear signatures of first- and second-order transitions within states of the same vorticity, which revealed the existence of different vortex configurations with the same vorticity. Schweigert and Peeters ${ }^{17}$ studied the field-cooled vortex states in mesoscopic superconducting disks and cylinders. They also calculated magnetization curves for different vortex configurations with the same total vorticity and found similar results as in Ref. 16.

This paper is organized as follows. In Sec. II the theoretical formalism is described. In Sec. III we discuss the different vortex states and in Sec. IV transitions between them are studied. In Sec. V we investigate larger disks and in Sec. VI we consider disks with finite thickness. In Sec. VII we compare our results with those obtained from the London approximation. Finally, in Sec. VIII we summarize our results. 


\section{THEORETICAL FORMALISM}

In the present paper, we consider thin superconducting disks immersed in an insulating medium in the presence of a perpendicular uniform magnetic field $H_{0}$. To solve this problem we follow the numerical approach of Schweigert and Peeters. ${ }^{5,6}$ As for thin disks $(d \ll \xi, \lambda)$ it is allowed to average the Ginzburg-Landau (GL) equations over the disk thickness. Using dimensionless variables and the London gauge $\operatorname{div} \vec{A}$ $=0$ for the vector potential $\vec{A}$, we write the system of GL equations in the following form:

$$
\begin{gathered}
\left(-i \vec{\nabla}_{2 D}-\vec{A}\right)^{2} \Psi=\Psi\left(1-|\Psi|^{2}\right), \\
-\Delta_{3 D} \vec{A}=\frac{d}{\kappa^{2}} \delta(z) \vec{j}_{2 D},
\end{gathered}
$$

where

$$
\vec{j}_{2 D}=\frac{1}{2 i}\left(\Psi * \vec{\nabla}_{2 D} \Psi-\Psi \vec{\nabla}_{2 D} \Psi *\right)-|\Psi|^{2} \vec{A}
$$

is the density of superconducting current. The superconducting wave function satisfies the boundary conditions $\left(-i \vec{\nabla}_{2 D}\right.$ $-\vec{A})\left.\Psi\right|_{n}=0$ normal to the sample surface and $\vec{A}=\frac{1}{2} H_{0} \rho \vec{e}_{\phi}$ far away from the superconductor. Here the distance is measured in units of the coherence length $\xi$, the vector potential in $c \hbar / 2 e \xi$, and the magnetic field in $H_{c 2}=c \hbar / 2 e \xi^{2}$ $=\kappa \sqrt{2} H_{c}$. The superconductor is placed in the $(x, y)$ plane, the external magnetic field is directed along the $z$ axis, and the indices 2D, 3D refer to two- and three-dimensional operators, respectively.

To solve the system of Eqs. (1) and (2), we used the approach of Ref. 6 for circular disks. We apply a finitedifference representation for the order parameter and the vector potential on a uniform Cartesian space grid $(x, y)$, with typically $128 \times 128$ grid points for the area of the superconductor, and use the link variable approach, ${ }^{18}$ and an iteration procedure based on the Gauss-Seidel technique to find $\Psi$. The vector potential is obtained with the fast Fouriertransform technique where we set $\vec{A}_{|x|=R_{S},|y|=R_{S}}=H_{0}(x$, $-y) / 2$ at the boundary of a box with a larger space grid of size typically four times the superconductor area.

For circular configurations such as disks the giant vortex state is characterized by the total angular momentum $L$ through $\Psi=\psi(\rho) \exp (i L \phi)$, where $\rho$ and $\phi$ are the cylindrical coordinates. $L$ is the winding number and gives the vorticity of the system. Due to the nonlinearity of the GL equations an arbitrary superconducting state is generally a mixture of different angular harmonics $L$ even in axially symmetric systems. Nevertheless, we can introduce an ana$\log$ to the total angular momentum $L$ which is still a good quantum number and which is in fact nothing else but the number of vortices in the system.

To find the different vortex configurations, which include the metastable states, we search for the steady-state solutions of Eqs. (1) and (2) starting from different randomly generated initial conditions. Then we increase/decrease slowly the

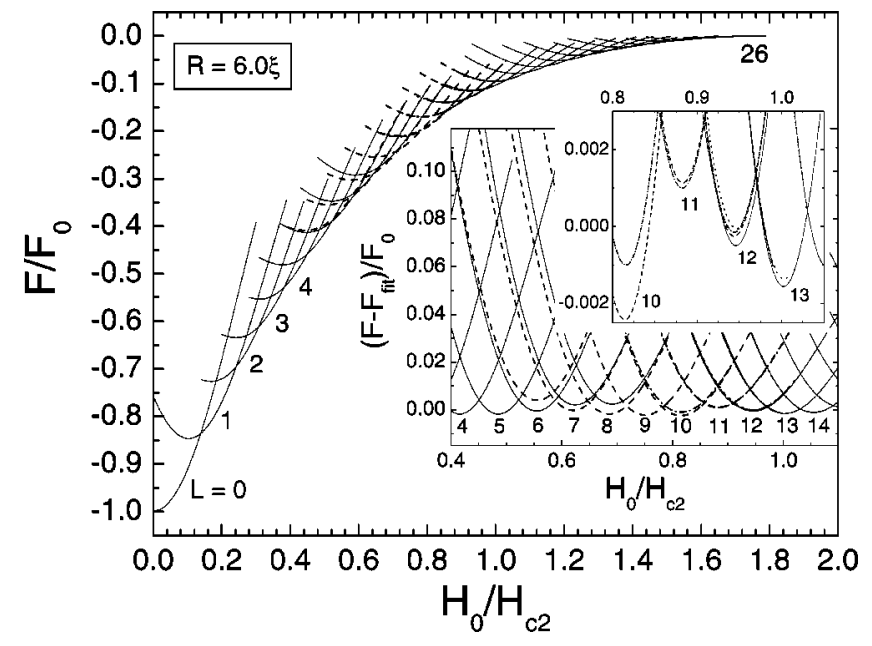

FIG. 1. The free energy of the different vortex configurations as a function of the applied magnetic field $H_{0}$. In the inset a quadratic fit, i.e., $F_{f i t} / F_{0}=-1.03496+1.547848 H_{0} / H_{c 2}$ $-0.61536\left(H_{0} / H_{c 2}\right)^{2}$ has been subtracted from the free energies of the different states. The free energy is in units of $F_{0}=H_{c}^{2} V / 8 \pi$.

magnetic field and recalculate each time the exact vortex structure. We do this for each vortex configuration in a magnetic-field range where the number of vortices stays the same. By comparing the dimensionless Gibbs free energies of the different vortex configurations

$$
F=V^{-1} \int_{V}\left[2\left(\vec{A}-\vec{A}_{0}\right) \cdot \vec{j}_{2 D}-|\Psi|^{4}\right] d \vec{r}
$$

where integration is performed over the sample volume $V$ and $\vec{A}_{0}$ is the vector potential of the uniform magnetic field, we find the ground state.

\section{THE DIFFERENT VORTEX STATES}

First we will concentrate on extremely thin superconducting disks, such that only the first GL equation [Eq. (1)] has to be solved and the magnetic field can be assumed to be homogeneous and equal to the external field, also inside the sample. Later, in Sec. VI, we will briefly consider disks with finite thickness and investigate the influence of such nonzero thickness on the stability of the different vortex configurations.

The free energy of the different vortex states in a superconducting disk with radius $R=6.0 \xi$ and $d \ll \lambda, \xi$ is given in Fig. 1. Contrary to the situation of smaller disks (see, for example, Ref. 6), now different vortex configurations with the same vorticity can nucleate. The inset shows the region where different vortex configurations with the same vorticity are possible in more detail. In an effort to make the picture more clear we approached the ground state in this region by a quadratic fit, i.e., $F_{f i t} / F_{0}=-1.03496+1.547848 H_{0} / H_{c 2}$ $-0.61536\left(H_{0} / H_{c 2}\right)^{2}$, and subtracted this energy from the free energies of the different states. The following vortex states were found.

(i) The $L=0$ state or the Meissner state, and the $L=1$ state where a single vortex is nucleated in the center of the 

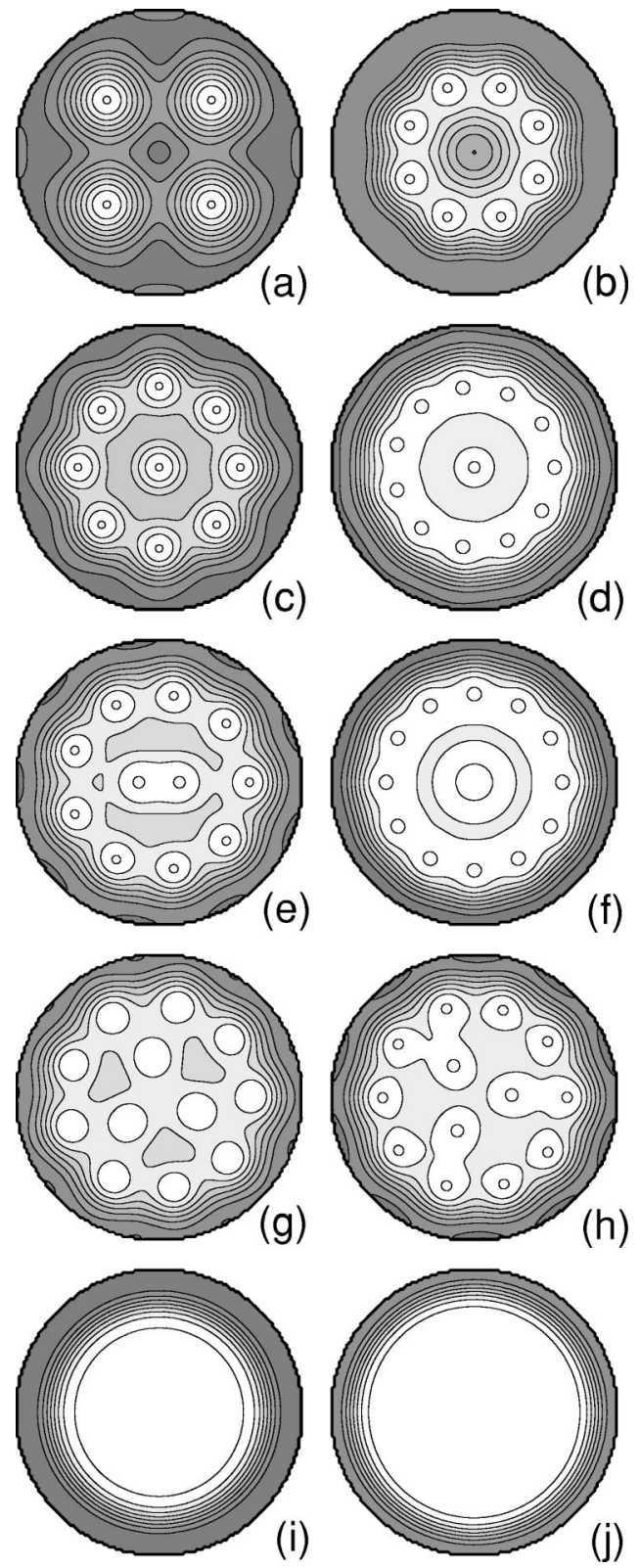

FIG. 2. Contour plots of the Cooper-pair density of the (4)-state at $H_{0} / H_{c 2}=0.3$ (a), the (8)-state at $H_{0} / H_{c 2}=0.7(\mathrm{~b})$, the $(1,8)$ state at $H_{0} / H_{c 2}=0.7(\mathrm{c})$, the $(1,11)$-state at $H_{0} / H_{c 2}=0.8(\mathrm{~d})$, the (2,9)-state at $H_{0} / H_{c 2}=0.8(\mathrm{e})$, the $\left(2_{g}, 12\right)$-state at $H_{0} / H_{c 2}=0.93$ (f), the $(3,9)$-state at $H_{0} / H_{c 2}=0.82(\mathrm{~g})$, the $(3,10)$-state at $H_{0} / H_{c 2}=0.88(\mathrm{~h})$, the 15 -state at $H_{0} / H_{c 2}=1.2(\mathrm{i})$, and the 24-state at $H_{0} / H_{c 2}=1.6(\mathrm{j})$. Dark (light) regions correspond to high (low) Cooper-pair density.

disk. The notations that we will use are 0-state and 1-state, respectively. The energy of these states are shown by solid curves in Fig. 1.

(ii) The multivortex state with vorticity $L$ where all $L$ vortices are separated and located on one shell (ring) which occurs for vorticity $L=2-8$. Notation: $(n)$-state with $n=L$ (the solid curves in Fig. 1). As an example the contour plots of the Cooper-pair density for the (4)-state and the (8)-state are shown in Figs. 2(a) and 2(b). Dark (light) regions correspond to high (low) Cooper-pair density. (iii) The multivortex state with vorticity $L$ with one vortex in the center while all the other vortices are positioned on a single shell around the center can be stable for $L=6-11$. Notation: $(1, n)$-state with $n=L-1$ (dashed curves in Fig. $1)$. The Cooper-pair density for the $(1,8)$-state and the (1,11)-state are given in Figs. 2(c) and 2(d).

(iv) The multivortex state with vorticity $L$ with two vortices on an inner shell and the other vortices on an outer shell can nucleate for $L=10-14$ (the dash-dotted curves in the inset of Fig. 1). Notation: $(2, n)$-state with $n=L-2$. When the two vortices in the center are combined into a giant vortex state with vorticity 2 , we use the notation $\left(2_{g}, n\right)$. Figures 2(e) and 2(f) show the Cooper-pair density of the $(2,9)$-state and the $\left(2_{g}, 12\right)$-state.

(v) The multivortex state with vorticity $L$ with three vortices on an inner shell and the other vortices on an outer shell can be stable for $L=12-14$ (see the dotted curves in the inset of Fig. 1). Notation: $(3, n)$-state with $n=L-3$. The Cooperpair density for the $(3,9)$-state and the $(3,10)$-state is given in Figs. 2(g) and 2(h).

(vi) The giant vortex state with vorticity $L$ where all the $L$ vortices are combined into one larger vortex in the center is possible for $L=12-24$ (solid curves in Fig. 1). Notation: $n$-state with $n=L$. As an example, the Cooper-pair density of the 15-state and the 24-state is shown in Figs. 2(i) and 2(j).

Notice that the difference in free energy between states with different vorticity is much larger than the free-energy difference between vortex states with the same vorticity but different vortex arrangements.

An overview of all the possible vortex configurations in a disk with radius $R=6.0 \xi$, their stability regions, and the regions over which they are the ground state are given in Table I. With increasing field the ground state changes as follows with increasing field: $0 \rightarrow 1 \rightarrow(2) \rightarrow(3) \rightarrow(4) \rightarrow(5) \rightarrow$ (6) $\rightarrow(1,6) \rightarrow(1,7) \rightarrow(1,8) \rightarrow(1,9) \rightarrow(2,9) \rightarrow 12 \rightarrow 13 \rightarrow 14 \rightarrow \cdots$ $\rightarrow 25 \rightarrow 26 \rightarrow$ normal state. The magnetic-field regions over which the different states correspond to a local minimum in the free energy, i.e., the magnetic-field region over which the different states are stable, are given in Fig. 3(a). Notice that for each type of structure there is an optimum $L$ value for which the structure is most stable. Figure 3(b) shows the magnetic-field region over which the state with vorticity $L$ is a ground state, i.e., corresponds to the global minimum in the free energy.

Figure 2(f) shows the Cooper-pair density of the $\left(2_{g}, 12\right)$-state, which has a giant vortex with vorticity 2 in the center. That this is indeed a giant vortex in the center can also be seen from Fig. 4 where the phase of the order parameter is shown for the same configuration. Phases near zero $(2 \pi)$ are given by light (dark) gray. Going around the central vortex the phase jumps two times with $2 \pi$, which means that the central vortex has vorticity $L=2$. This combination of a giant vortex state in the center and separated vortices around it has previously been found for smaller superconducting samples with noncircular geometries (i.e., squares and triangles), but for disks with radii $R \leqslant 4.0 \xi$ this combination was unstable. ${ }^{19}$ For larger disks (i.e., $R=6.0 \xi$ in our case) the new vortex configuration is stabilized.

At high magnetic fields, only giant vortex states are 
TABLE I. All possible (meta)stable vortex configurations for a disk with $R=6.0 \xi$ and $d \ll \lambda, \xi$. The first column gives the total vorticity, the second the vortex configuration, i.e., the distribution of the vortices over the different shells. $H_{e}, H_{p}$, and $\Delta H$ are, respectively, the expulsion field, the penetration field, and the magnetic-field range over which the considered vortex state can nucleate. $H_{g, e}, H_{g, p}$, and $\Delta H_{g}$ are the ground-state expulsion field, the ground-state penetration field, and the magnetic-field range over which the considered vortex state is the ground state.

\begin{tabular}{|c|c|c|c|c|c|c|c|}
\hline$L$ & Configuration & $H_{e} / H_{c 2}$ & $H_{p} / H_{c 2}$ & $\Delta H / H_{c 2}$ & $H_{g, e} / H_{c 2}$ & $H_{g, p} / H_{c 2}$ & $\Delta H_{g} / H_{c 2}$ \\
\hline 0 & 0 & 0.00 & 0.30 & 0.30 & 0 & 0.138 & 0.138 \\
\hline 1 & 1 & 0.00 & 0.39 & 0.39 & 0.138 & 0.242 & 0.104 \\
\hline 2 & (2) & 0.14 & 0.44 & 0.30 & 0.242 & 0.308 & 0.066 \\
\hline 3 & (3) & 0.20 & 0.51 & 0.31 & 0.308 & 0.383 & 0.075 \\
\hline 4 & (4) & 0.28 & 0.59 & 0.31 & 0.383 & 0.452 & 0.069 \\
\hline 5 & (5) & 0.31 & 0.67 & 0.36 & 0.452 & 0.522 & 0.070 \\
\hline 6 & $(6)$ & 0.37 & 0.74 & 0.37 & 0.522 & 0.586 & 0.064 \\
\hline 6 & $(1,5)$ & 0.39 & 0.74 & 0.35 & & & \\
\hline 7 & $(7)$ & 0.43 & 0.81 & 0.38 & & & \\
\hline 7 & $(1,6)$ & 0.45 & 0.81 & 0.36 & 0.586 & 0.648 & 0.062 \\
\hline 8 & $(8)$ & 0.48 & 0.87 & 0.39 & & & \\
\hline 8 & $(1,7)$ & 0.51 & 0.87 & 0.36 & 0.648 & 0.715 & 0.067 \\
\hline 9 & $(1,8)$ & 0.56 & 0.93 & 0.37 & 0.715 & 0.779 & 0.064 \\
\hline 10 & $(1,9)$ & 0.62 & 0.98 & 0.36 & 0.779 & 0.858 & 0.079 \\
\hline 10 & $(2,8)$ & 0.66 & 0.91 & 0.25 & & & \\
\hline 11 & $(1,10)$ & 0.68 & 1.03 & 0.35 & & & \\
\hline 11 & $(2,9)$ & 0.74 & 1.03 & 0.29 & 0.858 & 0.908 & 0.050 \\
\hline 12 & $(1,11)$ & 0.73 & 1.02 & 0.29 & & & \\
\hline 12 & $(2,10)$ & 0.80 & 1.01 & 0.21 & & & \\
\hline 12 & $(3,9)$ & 0.77 & 1.00 & 0.23 & & & \\
\hline 12 & 12 & 0.82 & 1.12 & 0.30 & 0.908 & 0.968 & 0.060 \\
\hline 13 & $(1,12)$ & 0.78 & 0.99 & 0.21 & & & \\
\hline 13 & $(2,11)$ & 0.84 & 0.99 & 0.15 & & & \\
\hline 13 & $(3,10)$ & 0.84 & 1.01 & 0.17 & & & \\
\hline 13 & 13 & 0.85 & 1.18 & 0.33 & 0.968 & 1.031 & 0.063 \\
\hline 14 & $(1,13)$ & 0.84 & 0.96 & 0.12 & & & \\
\hline 14 & $(2,12)$ & 0.89 & 0.928 & 0.038 & & & \\
\hline 14 & $\left(2_{g}, 12\right)$ & 0.928 & 0.98 & 0.052 & & & \\
\hline 14 & $(3,11)$ & 0.90 & 1.03 & 0.13 & & & \\
\hline 14 & 14 & 0.90 & 1.23 & 0.33 & 1.031 & 1.090 & 0.059 \\
\hline 15 & 15 & 0.91 & 1.29 & 0.38 & 1.090 & 1.151 & 0.061 \\
\hline 16 & 16 & 0.93 & 1.35 & 0.42 & 1.151 & 1.210 & 0.059 \\
\hline 17 & 17 & 1.02 & 1.41 & 0.39 & 1.210 & 1.269 & 0.059 \\
\hline 18 & 18 & 1.08 & 1.46 & 0.38 & 1.269 & 1.328 & 0.059 \\
\hline 19 & 19 & 1.17 & 1.51 & 0.34 & 1.328 & 1.387 & 0.059 \\
\hline 20 & 20 & 1.21 & 1.53 & 0.32 & 1.387 & 1.450 & 0.063 \\
\hline 21 & 21 & 1.29 & 1.59 & 0.30 & 1.450 & 1.510 & 0.060 \\
\hline 22 & 22 & 1.38 & 1.65 & 0.27 & 1.510 & 1.571 & 0.061 \\
\hline 23 & 23 & 1.44 & 1.67 & 0.23 & 1.571 & 1.621 & 0.050 \\
\hline 24 & 24 & 1.55 & 1.73 & 0.21 & 1.621 & 1.687 & 0.066 \\
\hline 25 & 25 & 1.61 & 1.77 & 0.16 & 1.687 & 1.732 & 0.045 \\
\hline 26 & 26 & 1.69 & 1.79 & 0.10 & 1.732 & 1.790 & 0.058 \\
\hline
\end{tabular}

stable. In this case all the vortices collapse into the center to form a giant vortex state which corresponds with a singular zero in the Cooper-pair density. Since we solve the Ginzburg-Landau equations numerically, we took the following convention: when the highest Cooper-pair density in the region between two vortices is less than $10^{-4}$, we consider these vortices as combined into a giant vortex. Figure 5 shows the contour plot of the logarithm of the Cooper-pair density for the giant vortex state with $L=13$ at $H_{0} / H_{c 2}$ $=1.0$. Using a logarithmic scale for the Cooper-pair density 


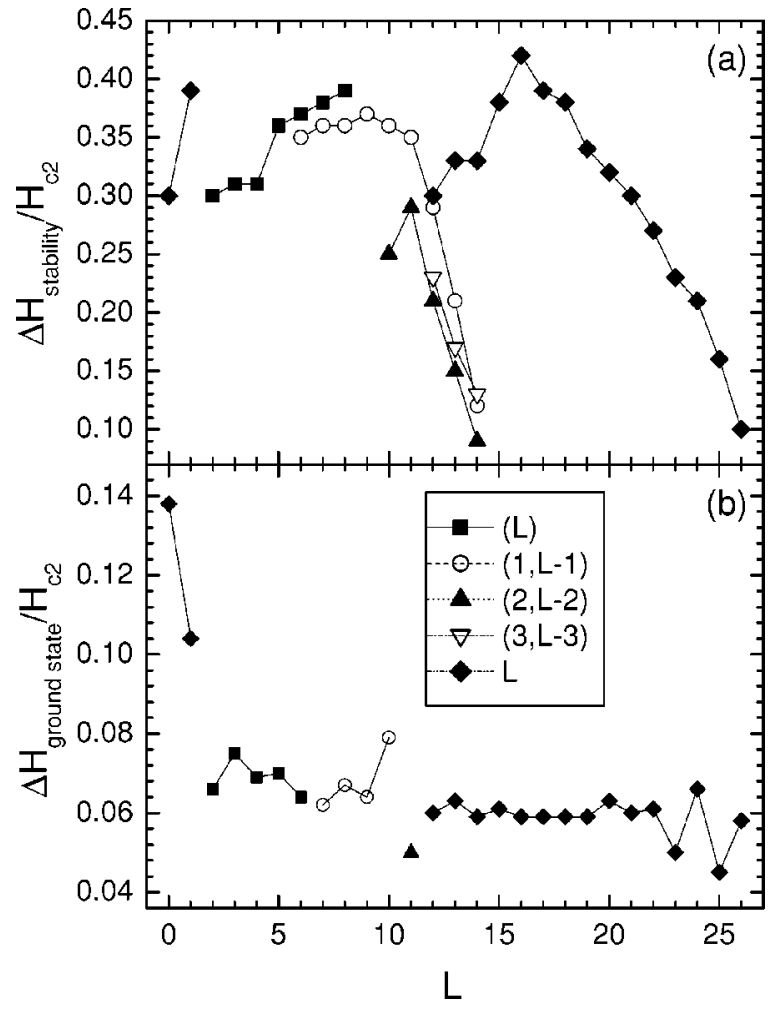

FIG. 3. (a) The magnetic-field regions over which the different vortex states are stable, i.e., corresponding to a local minimum in the free energy, and (b) the magnetic-field regions over which the states are the ground state, i.e., corresponding to the global minimum in the free energy.

we see clearly 13 vortices situated on a single ring, but the maximum Cooper-pair density between two separate vortices is of the order of $10^{-6}-10^{-8}$ (which will not be discernible experimentally) and therefore we consider the state as a giant vortex state. We found the same fine structure for the 12-state and the 14-state. In the case of the 12-state the maximum between two vortices is of the order of $10^{-5}-10^{-6}$, for the 14-state the maximum is of the order of $10^{-8}-10^{-10}$. For states with higher vorticities we do not find such a fine struc-

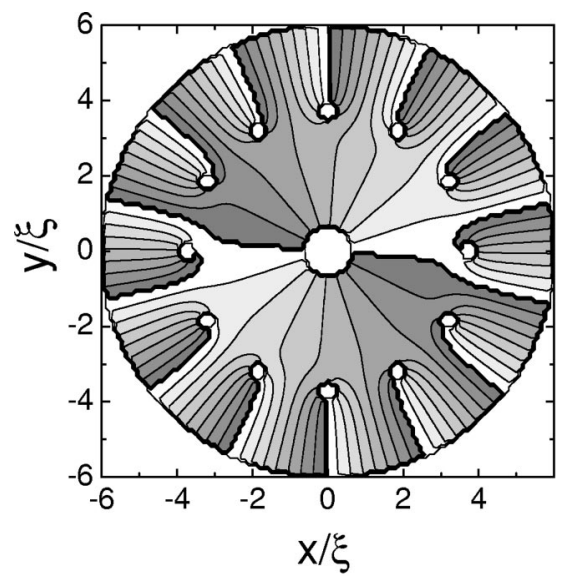

FIG. 4. The phase of the order parameter for the configuration of Fig. 2(f).

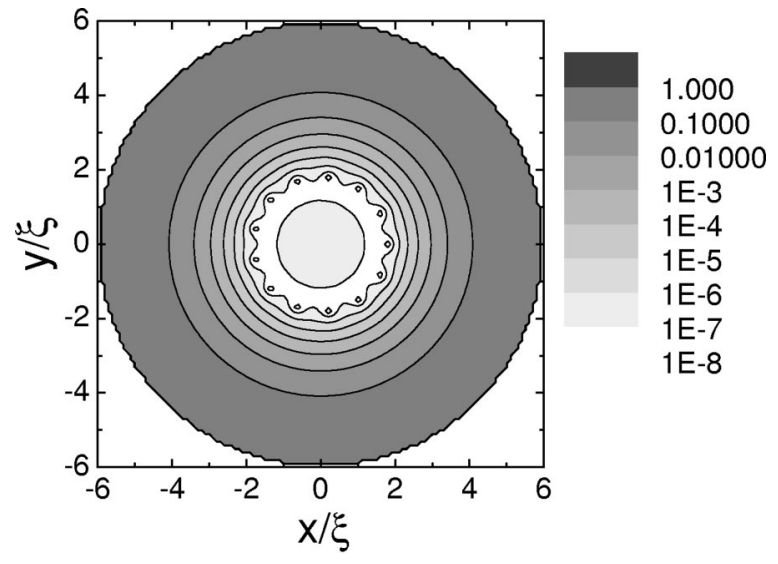

FIG. 5. The logarithm of the Cooper-pair density of the 13-state.

ture with our numerical procedure.

In Fig. 6 the magnetic moment $D$ is given as a function of the applied magnetic field, i.e.,

$$
\vec{D} / H_{c 2}=\int_{V} \vec{r} \times \vec{j}_{2 D} d \vec{r} / \int_{V} d \vec{r}
$$

where integration is performed over the sample volume $V$. The magnetic moments of the different vortex states are given by the same type of curves as their free energy in Fig. 1. The solid vertical lines indicate the ground-state transitions. Notice that also the difference in magnetic moment between states with the same vorticity is much smaller than the one between states with a different vorticity. In the inset the symbols show the jump in magnetic moment for the ground-state transitions, which can be fitted to the curve $\Delta D / H_{c 2}=(a+b L) /(1+c L)$ with $a=0.748, \quad b=-0.022$, and $c=0.249$. The fitted curve is given by the solid curve in the inset of Fig. 6. Notice that $\Delta D$ monotonically decreases with increasing vorticity, except for $L=10$. For $L=10$ the ground state transits from the configuration with one vortex in the center to one in which we have two vortices in the center (which is only a ground state for $L=11$ ). For $L=12$

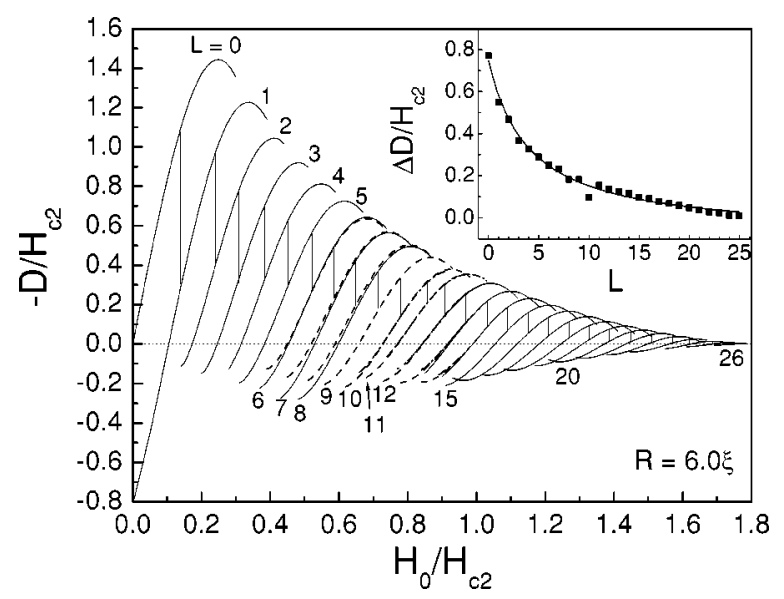

FIG. 6. The magnetic moment of the different vortex states in a disk with radius $R=6.0 \xi$ as a function of the applied magnetic field. The inset shows the jump in magnetic moment, when following the ground state. 


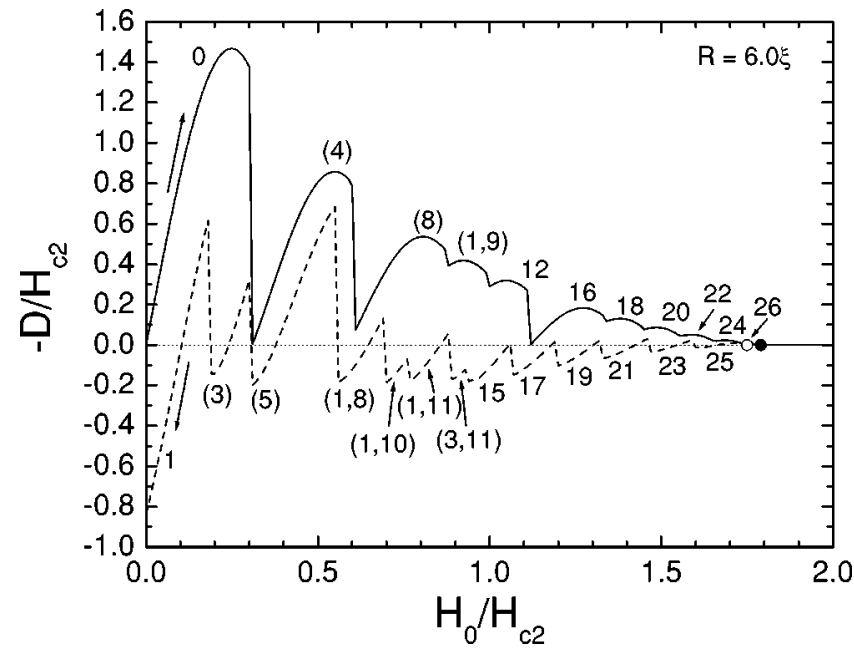

FIG. 7. The magnetic moment as a function of the applied magnetic field for increasing field (solid curve) and decreasing field (dashed curve). The solid (open) dot indicates the superconducting/ normal transition field with increasing (decreasing) magnetic field.

we immediately have a giant vortex in the center. Consequently, the $L=11$ configuration with two vortices in the center is not very stable, which is also seen in Fig. 3(b).

\section{TRANSITIONS BETWEEN VORTEX STATES}

Next we investigated the transitions between different vortex states. While in smaller disks the vorticity always changes with one unit at the penetration field ${ }^{6}$ the situation in larger disks is more complicated. Now, it becomes possible that several vortices will enter/leave the disk at the transition fields. With increasing magnetic field, the superconducting state for an ideal disk evolves as $0 \rightarrow(4) \rightarrow(8) \rightarrow(1,9) \rightarrow 12 \rightarrow 16 \rightarrow 18 \rightarrow 20 \rightarrow 22 \rightarrow 24 \rightarrow 26$ $\rightarrow$ normal state, and with decreasing field as normal state $\quad \rightarrow 25 \rightarrow 23 \rightarrow 21 \rightarrow 19 \rightarrow 17 \rightarrow 15 \rightarrow(3,11) \rightarrow(1,11)$ $\rightarrow(1,10) \rightarrow(1,8) \rightarrow(5) \rightarrow(3) \rightarrow 1$. The corresponding magnetic moment is given in Fig. 7 as a function of the applied magnetic field for increasing (solid curve) and decreasing field (dashed curve). When more vortices enter/leave during a transition the jump in magnetic moment is larger than when the vorticity changes only by one unit. The solid (open) dot indicates the superconducting/normal transition field with increasing (decreasing) field.

With increasing field the Meissner state transits into the (4)-state, instead of going into the one-vortex state as would be the case in smaller disks. Figure 8 shows the evolution of the free energy during this transition from the Meissner state to the (4)-state for $H_{0} / H_{c 2} \approx 0.3$, when the Meissner state is no longer stable. The insets show the Cooper-pair density of the vortex state during the transition at specific iteration steps indicated by the symbols in the figure. During the first 400 numerical iterations the state and its free energy change only slightly. Inset (a) clearly shows that after 420 iteration steps the state still contains no vortices, i.e., $L=0$. Around the 470th iteration step the free energy starts decreasing faster. The contour plot (b) of the Cooper-pair density shows four

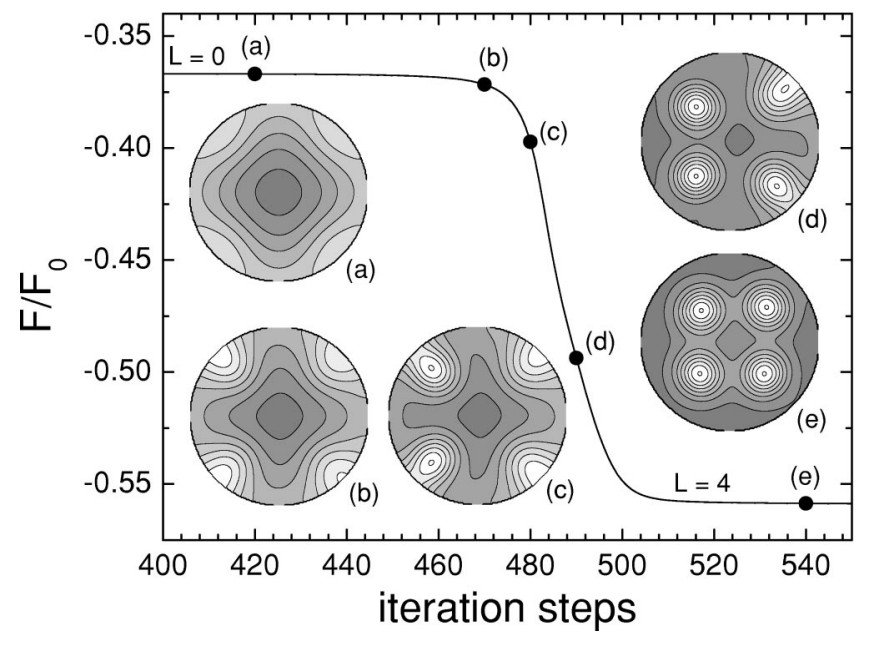

FIG. 8. The evolution of the free energy during the transition from the Meissner state to the (4)-state. The insets show the contour plots of the Cooper-pair density at the iteration steps indicated by the symbols in the main figure.

spots along the boundary with a local decrease of the Cooper-pair density. Further evolving to a stable state, the free energy decreases and first two vortices enter the superconducting disk [inset (c)], later two other vortices enter the disk [inset (d)], and finally the state with one shell of four vortices stabilizes [inset (e)]. Approximately another 1500 iteration steps are needed to converge completely to the stable state with $L=4$. In this regime the vortex state and its free energy change only slightly.

We mentioned above that the state in which all $L$ vortices are located on a single shell are stable for $L=2-8$. We also tried to find the (9)-state with nine vortices on one shell, but this state was unstable for the considered parameters. Figure 9 shows the evolution of the free energy as function of the number of numerical iterations in finding a stable state with $L=9$. The insets show the Cooper-pair density at the points

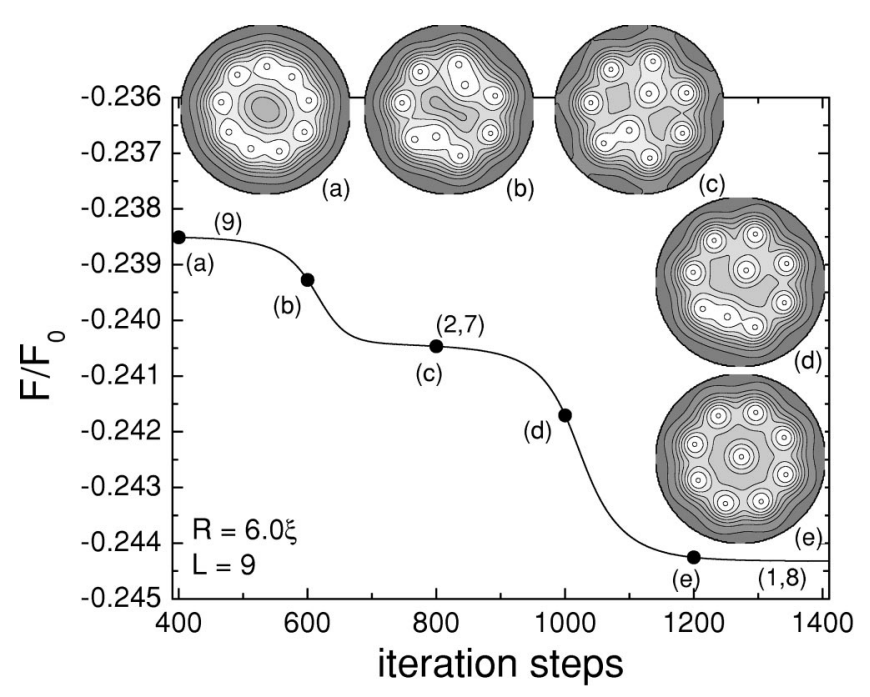

FIG. 9. The evolution of the free energy during the transition from the $(9)$-state to the $(1,8)$-state. The insets show the contour plots of the Cooper-pair density. 


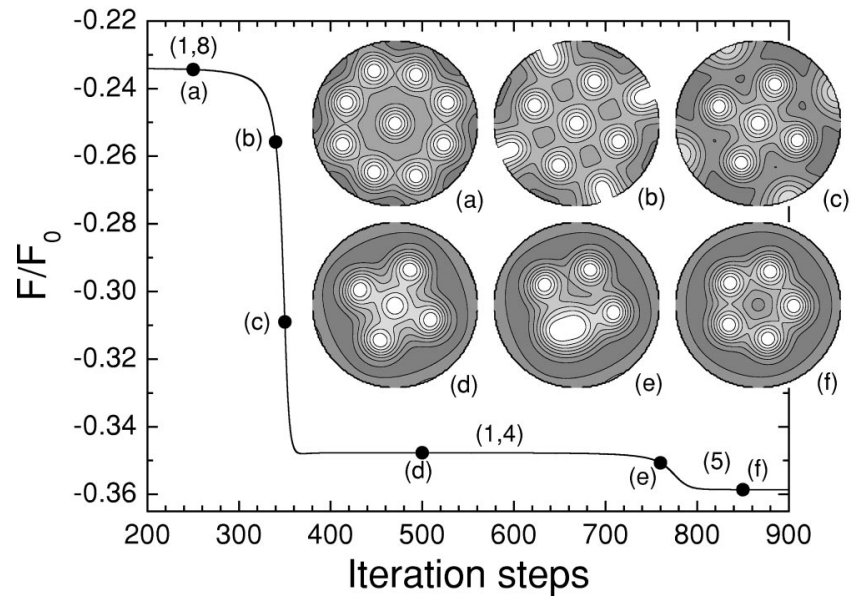

FIG. 10. The evolution of the free energy during the transition from the $(1,8)$-state to the $(5)$-state. The insets show the contour plots of the Cooper-pair density.

indicated by the symbols. We started our computer program with a vortex configuration in which nine vortices are located on a single ring. After 400 iteration steps the state contains still nine vortices on one (deformed) shell [inset (a)]. Then, the free energy starts decreasing faster and two vortices move towards the center [insets (b) and (c)]. The program tries to find a stable state with two vortices on an inner shell and the other vortices on the outer shell, i.e., the $(2,7)$-state, but this state is also unstable. Therefore, after more iteration steps the free energy decreases again and one of the two vortices from the inner shell moves towards the outer one [inset (d)] and finally the program converges to the $(1,8)$ state with one vortex in the center and eight vortices on a shell around it [inset (e)].

Next we investigate the expulsion of vortices. As an example, we consider the transition from the $(1,8)$-state to the (5)-state which occurs at $H_{0} / H_{c 2}=0.56$ with decreasing field. Figure 10 shows the free-energy evolution during the transition as a function of the iteration steps. The insets show the Cooper-pair density at the fields indicated by the symbols. After 250 iteration steps the state still contains one vortex in the center and eight vortices on a (slightly deformed) shell around the vortex in the center [inset (a)]. Then, with increasing iteration steps, the free energy decreases when four vortices from the outer shell leave the superconductor [insets $(\mathrm{b}, \mathrm{c})]$. Then, the program tries to find a stable $(1,4)$-state $[$ inset $(d)]$, but, since this state does not correspond to a local minimum in the free energy, the state evolves into a state with a lower energy [inset (e)], i.e., the vortex from the center moves to the outer shell. Finally the program finds a stable (5)-state with five vortices on a single shell [inset (f)].

Above we showed that the $\left(2_{g}, 12\right)$-state, with a giant vortex state with vorticity $L=2$ in the center and 12 vortices around it on one shell, is stable at $H_{0} / H_{c 2}=0.93$. With decreasing magnetic field this state continuously transits into a state with two separate vortices in the center, i.e., the $(2,12)$-state. This is shown in Fig. 11, which gives the free energy for this state and the insets are contour plots of the Cooper-pair density at the fields indicated by the symbols. At

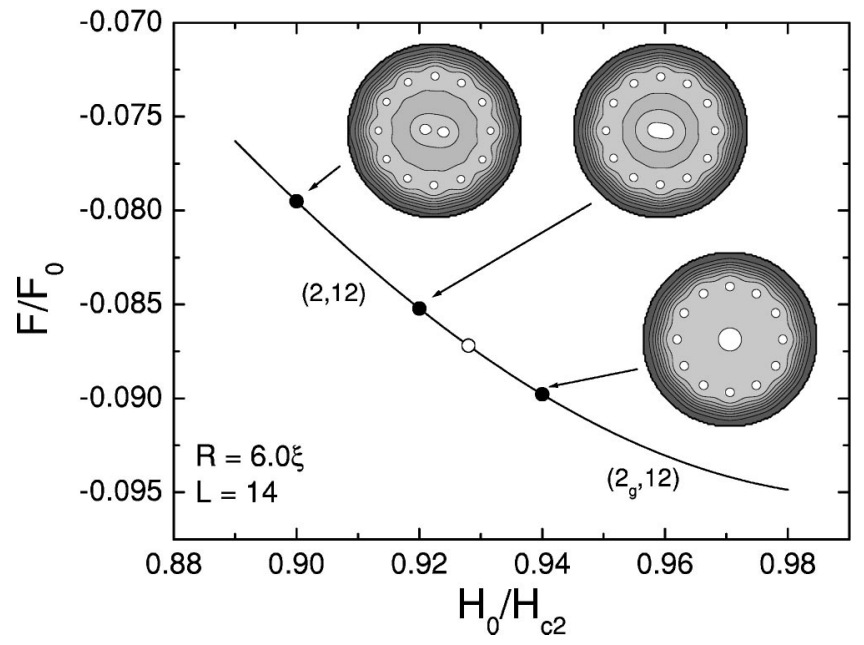

FIG. 11. The free energy of the $(2,12)$-state and the $\left(2_{g}, 12\right)$-state as a function of the applied magnetic field. The insets show the contour plots of the Cooper-pair density at the fields indicated by the closed symbols. The open symbol indicates the field where the two central vortices recombine in the center into a giant vortex with vorticity $L=2$.

low fields we clearly find two separate vortices on an inner shell and 12 vortices on an outer one. With increasing field, the two inner vortices move continuously towards the center where they combine into a giant vortex state with vorticity $L=2$ at the field indicated by the open symbol in Fig. 11 .

\section{LARGER DISKS}

Up to now, we considered superconducting disks with radius equal to $R=6.0 \xi$. In larger superconducting disks one expects more shells of vortices and also more possible stable vortex configurations for a fixed number of vortices. As an example we consider disks with radius $R=20 \xi$ for the case of 16 vortices. At the applied magnetic field $H_{0}=0.1 H_{c 2}$ we find three different vortex configurations with vorticity $L$ $=16$, i.e., the $(5,11)$-state, the $(4,12)$-state, and the $(1,5,10)$-state. The free energies of these states as a function of the applied magnetic field are shown in Fig. 12. The $(1,5,10)$-state has the lowest energy of the three states over their whole region of stability and the $(4,12)$-state the highest one. Notice that the curves do not cross each other. The Cooper-pair densities for the three states at the applied magnetic field $H_{0}=0.1 H_{c 2}$ are given in the insets of Fig. 12 .

The distributions of vortices are very similar to the distributions of particles in classical two-dimensional atoms. ${ }^{2}$ Depending on the confinement potential and the particle interaction the lowest-energy state with 16 particles can be the $(1,5,10)$-state or the $(5,11)$-state. ${ }^{20}$

\section{DISKS WITH FINITE THICKNESS}

In this section we investigate the effect of the nonzero thickness of the superconducting disks on the stability of the vortex states and the transition fields. In this case we cannot neglect the second Ginzburg-Landau equation (2) that describes the bending of the magnetic field around the super- 


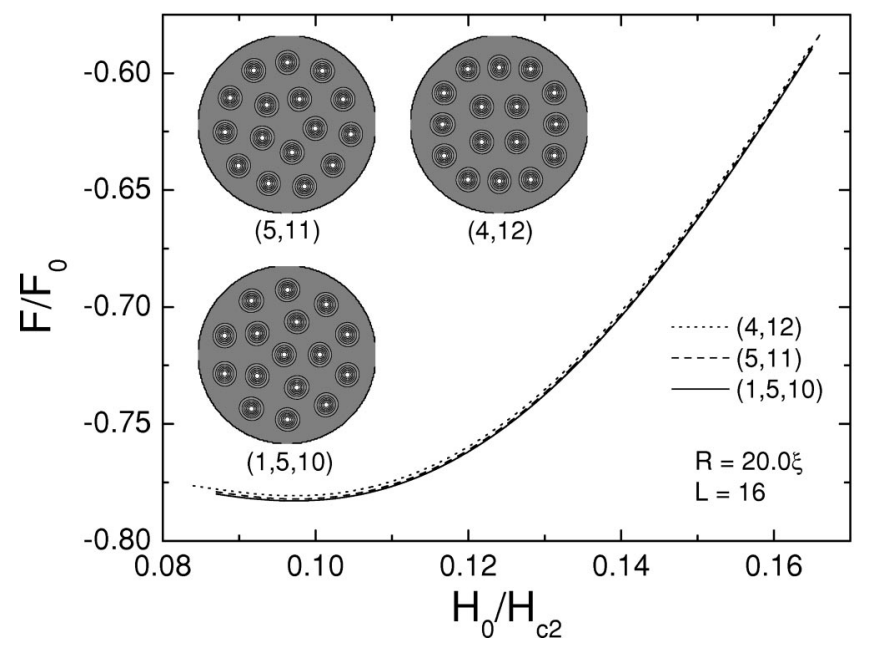

FIG. 12. The free energy as a function of the applied magnetic field for the $(4,12)$-state, the $(5,11)$-state, and the $(1,5,10)$-state in a superconducting disk with radius $R=20 \xi$. The insets show the contour plots of the Cooper-pair density for these states at $H_{0} / H_{c 2}$ $=0.1$

conductor. This means that we have to solve the complete set of nonlinear Ginzburg-Landau equations as described above.

As an example, we investigate the influence of the thickness on the stability of the vortex states with vorticity $L$ $=7$ in a superconducting disk with radius $R=6.0 \xi$. Figure 13 shows the free energy as a function of the applied magnetic field for the $(1,6)$-state (thick solid curves) and the (7)state (thick dashed curves) for different values of the thickness, i.e., $d=0.005 \xi$ (curves 1 ), $0.1 \xi$ (curves 2 ), and $0.2 \xi$ (curves 3 ). In the inset the free energy is shown for the case of a cylinder, i.e., $d=\infty$ (see also, e.g., Ref. 21). For $d$ $=0.005 \xi$ the $(1,6)$-state and the $(7)$-state are stable over almost the same magnetic-field region (see also Table II). With increasing thickness, we find that the magnetic-field region over which the two states are stable decreases. This effect is much more pronounced for the $(1,6)$-state than for the $(7)$ state. But notice that for a cylinder the states are stable over a larger magnetic-field region. The reason is that initially with increasing thickness $d$ more magnetic field is expelled which increases the magnetic-field pressure and which reduces the stability of the vortex state with increasing magnetic field. In the case of a cylinder there is no demagnetization effect and consequently this effect is not present. With increasing thickness also the free-energy difference decreases between states with the same total vorticity, but with different distributions of the vortices over the different shells.

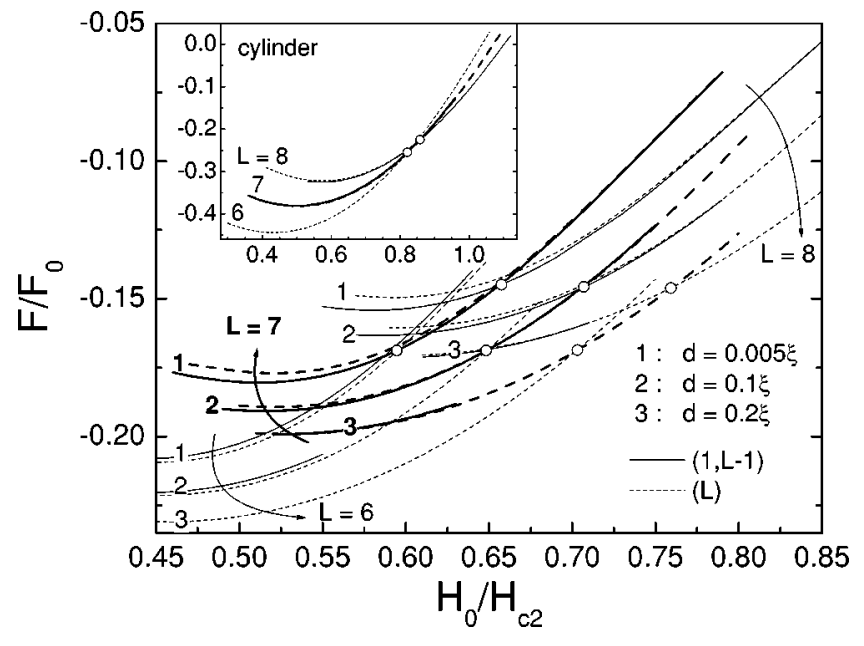

FIG. 13. The free energy of the different vortex configurations with vorticity $L=6,7,8$ as a function of the applied magnetic field for a superconducting disk with radius $R=6.0 \xi$ and thickness $d$ $=0.005 \xi, 0.1 \xi, 0.2 \xi$. The inset shows the free energy in the case of a cylinder, i.e., $d=\infty$. The open symbols indicate the ground-state transition fields.

Another remarkable influence of the sample thickness can be found by looking at the state with the lowest free energy as a function of the magnetic field. For $d=0.005 \xi$ the $(1,6)$-state has a lower energy compared to the (7)-state over the entire magnetic-field region where both states are stable. For $d$ $=0.1 \xi$ we see that the $(1,6)$-state has the lower energy for low magnetic fields, while for $H_{0} \gtrsim 0.65 H_{c 2}$ the (7)-state has the lowest energy. When we further increase the sample thickness to $d=0.2 \xi$ we see that the (7)-state has the lower energy over its whole stability region. This is also the case for the cylinder.

To investigate the influence of the sample thickness on the transition fields, we also calculated the different vortex states with vorticity $L=6$ and $L=8$ (see the thinner curves in Fig. 13). The penetration and the expulsion fields are indicated by the open symbols in the figure. It is immediately clear that the ground-state transition fields move to higher fields with increasing sample thickness. The magnetic-field region over which the $L=7$ state is the ground state decreases with increasing disk thickness (see also Table II). Notice further that the $(1,5)$-state is stable for samples with thickness $d$ $=0.005 \xi$ and $0.1 \xi$, but it becomes unstable for $d=0.2 \xi$ and the cylinder. Notice further that the free energy where the ground-state transitions occur is almost independent of the

TABLE II. The magnetic-field regions over which the (1,6)-state and the (7)-state are stable, i.e., $(\Delta H) / H_{c 2}$, and are the ground state, i.e., $\left(\Delta H_{g}\right) / H_{c 2}$, for different values of the sample thickness $d$.

\begin{tabular}{lcccc}
\hline \hline$d / \xi$ & $(\Delta H)_{(1,6)} / H_{c 2}$ & $(\Delta H)_{(7)} / H_{c 2}$ & $\left(\Delta H_{g}\right)_{(1,6)} / H_{c 2}$ & $\left(\Delta H_{g}\right)_{(7)} / H_{c 2}$ \\
\hline 0.005 & 0.33 & 0.32 & 0.0629 & \\
0.1 & 0.17 & 0.31 & & 0.0586 \\
0.2 & 0.11 & 0.29 & 0.0559 \\
$\infty$ & 0.96 & 1.09 & 0.0369 \\
\hline \hline
\end{tabular}


TABLE III. Similar as Table I but now for the results within the London approach where a cutoff at $\xi / 2$ was used.

\begin{tabular}{rcccc}
\hline \hline$L$ & Configuration & $H_{g, e} / H_{c 2}$ & $H_{g, p} / H_{c 2}$ & $\Delta H_{g} / H_{c 2}$ \\
\hline 0 & 0 & 0 & 0.138 & 0.138 \\
1 & 1 & 0.138 & 0.233 & 0.095 \\
2 & $(2)$ & 0.233 & 0.287 & 0.054 \\
3 & $(3)$ & 0.287 & 0.352 & 0.065 \\
4 & $(4)$ & 0.352 & 0.422 & 0.070 \\
5 & $(5)$ & 0.422 & 0.488 & 0.066 \\
6 & $(1,5)$ & 0.488 & 0.522 & 0.034 \\
7 & $(1,6)$ & 0.522 & 0.594 & 0.072 \\
8 & $(1,7)$ & 0.594 & 0.670 & 0.076 \\
9 & $(1,8)$ & 0.670 & 0.717 & 0.047 \\
10 & $(2,8)$ & 0.717 & 0.766 & 0.049 \\
11 & $(3,8)$ & 0.766 & 0.806 & 0.040 \\
12 & $(3,9)$ & 0.806 & 0.877 & 0.071 \\
13 & $(4,9)$ & 0.877 & 0.910 & 0.033 \\
14 & $(4,10)$ & 0.910 & 0.987 & 0.077 \\
15 & $(5,10)$ & 0.987 & 1.031 & 0.044 \\
\hline \hline
\end{tabular}

thickness for $d / \xi=0.005,0.1$, and 0.2 , while the transition field increases with increasing thickness.

\section{COMPARISON WITH THE LONDON APPROXIMATION}

Finally, we compare the vortex configurations obtained within the London approach and the previous nonlinear Ginzburg-Landau theory. We considered a thin disk $(d \ll R$ $\ll \Lambda=\lambda^{2} / d$ ) with $R=6.0 \xi$. In this limit demagnetization effects may be neglected and a close analogy between vortices and electrical charges can be made ${ }^{13}$ (see the Appendix for details of the calculation).

First, we obtained an expression for the energy of the system in the case of vortices distributed in two rings with radius $\rho_{1}$ (inner ring) and $\rho_{2}$ (outer ring), $F^{\text {rings }}$ [cf. Eq. (A10)]. This energy was minimized for several vortex configurations in a disk of radius $6.0 \xi$ as function of the magnetic field. For only a single ring with one possible vortex in the center, there is only one free parameter, the radius of the ring, $\rho_{1}$. For the case with two rings, there are the free variational parameters: $\rho_{1}, \rho_{2}$, and $\alpha$ (the misalignment angle between the two rings). In each of these two cases we minimized the energy $F^{\text {rings }}$ with respect to the free parameters. This procedure allows us to find stable vortex states for different number of vortices in the inner and outer shells, as well as the following ground-state configurations: $0 \rightarrow 1 \rightarrow(2) \rightarrow(3) \rightarrow(4) \rightarrow(5) \rightarrow(1,5) \rightarrow(1,6)$ $\rightarrow(1,7) \rightarrow(1,8) \rightarrow(2,8) \rightarrow(3,8) \rightarrow(3,9) \rightarrow(4,9) \rightarrow(4,10)$ $\rightarrow(5,10) \rightarrow(5,11) \cdots \quad$ (see Table III for the region over which each configuration is the ground state). Such configurations were already obtained for a thin disk with $R / \xi$ $=\exp (4)=54.60$ by Buzdin and Brison ${ }^{13}$ [but only up to the $(1,7)$-state], and for a cylinder with $R \gg \lambda$ by Venegas and Sardella. ${ }^{22}$ However, in our case, the ground-state transitions from one configuration to another strongly depend on the cutoff procedure adopted. For example, to obtain the values shown in Table III, instead of using the cutoff $i=j \rightarrow \mid \vec{\rho}_{i}$ $-\vec{\rho}_{j} \mid / \xi=1$, we employed a cutoff in which $i=j \rightarrow \mid \vec{\rho}_{i}$ $-\vec{\rho}_{j} \mid / \xi=1 / 2$ (see the Appendix). This yielded better defined transitions and results which are in better accordance with those obtained within the nonlinear Ginzburg-Landau theory at low fields. Nevertheless, the ground-state configurations start to differ from the ones predicted by the GinzburgLandau theory as soon as we have six vortices inside the disk (compare Tables I and III). For instance, the London approach gives the $(1,5)$-state as the ground state while the Ginzburg-Landau theory predicts the (6)-state. ${ }^{6}$ This difference is not due to the cutoff value adopted since it gives the same contribution whenever six vortices were present inside the disk. In fact, these discrepancies arise because within the London theory the vortices are pointlike objects and one has no information about the Cooper-pair density in the superconducting material. Therefore, these results suggest that, for $R=6.0 \xi$, a theory which takes into account the spatial dependence of the order parameter (Ginzburg-Landau theory) must be employed in order to obtain accurate results for $L$ $>5$.

To investigate in deeper detail the actual vortex configurations when two vortex shells are present inside the disk, the assumption of vortices distributed within two rings should be relaxed. Therefore, the vortex configurations inside the disk were also obtained by minimizing the free energy [Eq. (A9)] while considering each vortex position as a free parameter. We performed Monte Carlo (MC) simulations using typically $5 \times 10^{5}$ steps to thermalize the system and another $10^{6}$ steps to compute averages for each value of the magnetic field and vorticity $L$. The resulting ground states were the same as the ones calculated by minimizing the energy within the tworing approximation. For the states $\left(N_{1}\right)$ and $\left(1, N_{1}\right)$ the $\mathrm{MC}$ method yields basically the same energy values obtained from a minimization of $\mathcal{G}^{\text {rings }}[\mathrm{Eq}$. (A10)]. However, the vortex positions show some differences when two shells were present, since vortices in a shell are no longer distributed perfectly on a ring. Also, we obtained slightly lower-energy values.

Next, we compare the configurations obtained within the London approach with the results within the nonlinear Ginzburg-Landau theory in the case that both theories predicted the same vortex configurations. Figures 14(a) and 14(d) give the contour plots of the Cooper-pair density for the (4)-state at $H_{0} / H_{c 2}=0.30,(1,8)$-state at $H_{0} / H_{c 2}$ $=0.70,(2,8)$-state at $H_{0} / H_{c 2}=0.70$, and the $(3,9)$-state at $H_{0} / H_{c 2}=0.82$, respectively. The black dots are the positions of the vortices calculated within the London theory (it should be noticed that the choice of the cutoff value is not important in the calculation of the vortex positions, since its contribution to the energy is a constant value for given vorticity $L$ ). Although some ground-state configurations predicted by the London and the nonlinear Ginzburg-Landau theories were different, these figures show that the positions of the vortices are quite similar for some of the stable configurations. However, the positions of the vortex cores do not always coincide exactly as, for example, is clearly seen in Fig. 14(c) for the 

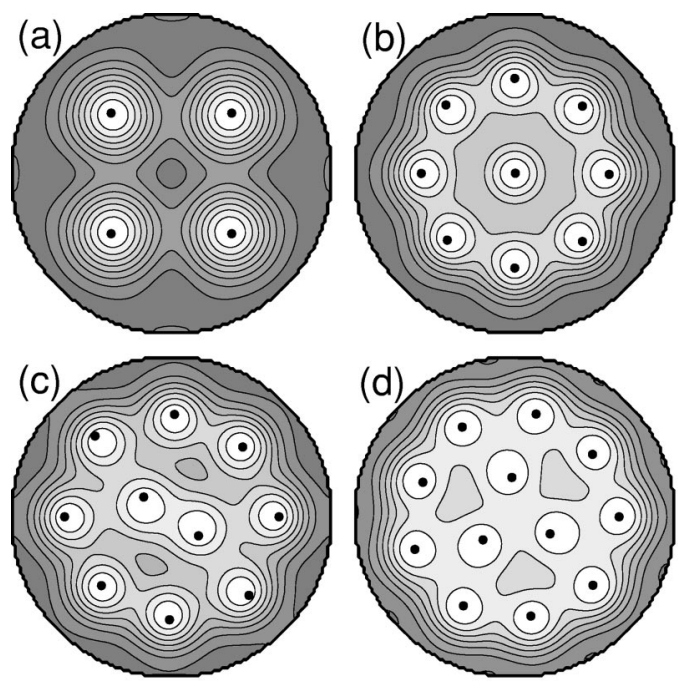

FIG. 14. Comparison of vortex positions obtained within the nonlinear Ginzburg-Landau theory (contour plot) and the London approach (solid dots). The stable configurations are the (4)-state at $H / H_{c 2}=0.30(\mathrm{a})$, the $(1,8)$-state at $H / H_{c 2}=0.70(\mathrm{~b})$, the $(2,8)$-state at $H / H_{c 2}=0.70(\mathrm{c})$, and the $(3,9)$-state at $H / H_{c 2}=0.82(\mathrm{~d})$.

$(2,8)$-state at $H_{0} / H_{c 2}=0.70$. On the other hand, sometimes the vortex cores do almost coincide in both calculations [see, for example, Figs. 14(a), 14(b), and 14(d)], but from the Ginzburg-Landau theory one obtains much more information about the density distribution of the superconducting electrons.

\section{CONCLUSIONS}

We studied the vortex configurations in superconducting disks with a sufficiently large radius such that several shells of vortices can be stabilized. We found that for fixed vorticity different distributions of vortices over the different shells are possible. However, the free-energy difference between those states is very small, compared to the difference between the free energy of vortex states with different vorticity. We made an overview of all stable and metastable vortex states in a superconducting disk with radius $R=6.0 \xi$ and indicated which vortex state has the lowest energy at a certain applied magnetic field.

Contrary to small disks, the change in vorticity at the transition field can be larger than one. With increasing field, the Meissner state, for example for $R=6 \xi$, transits directly into a state with four separate vortices. Also contrary to the small disk case, a combination of a giant vortex state and a multivortex state is possible. We investigated the evolution of the $(2,12)$-state where, with increasing field, the two vortices on the inner shell move to the center, combining there into a giant vortex.

We also examined the influence of the thickness of the disk on the vortex configuration and the transition fields. We found that with increasing thickness the stability region of the vortex states decreases, but in the limit of a cylinder (i.e., $d \rightarrow \infty)$ the stability region is substantially larger. Also the difference in free energy between different vortex states with the same total vorticity decreases. Moreover, by changing the sample thickness, it is possible that the lowest-energy state changes from one vortex configuration to another. The penetration and expulsion fields move to higher fields with increasing sample thickness and the magnetic-field region over which the vortex state is the ground state decreases.

Finally, we compared the vortex states calculated within the nonlinear Ginzburg-Landau and the London theories. We found that the ground states determined by the London approach are similar to the ones predicted by the nonlinear Ginzburg-Landau theory only for small vorticity $L$ (up to $L$ $<6$ for $R=6.0 \xi$ ). Also the difference in the region over which a given state is the ground-state configuration predicted by the London and nonlinear Ginzburg-Landau theories increases as the vorticity $L$ increases. Best accordance is achieved by using $i=j \rightarrow\left|\vec{\rho}_{i}-\vec{\rho}_{j}\right|=\xi / 2$ as the cutoff (in the London limit). However, we also found that vortex positions for some stable states calculated by using both theories were quite similar. This shows that, although the vortex positions may be calculated with relatively good accuracy for certain $L$ values, the applicability of the London approach to find the ground vortex states is restricted to small values of the vorticity $L$.

\section{ACKNOWLEDGMENTS}

This work was supported by the Flemish Science Foundation (FWO-Vl), the "Onderzoeksraad van de Universiteit Antwerpen" (GOA), the "Interuniversity Poles of Attraction Program-Belgian State, Prime Minister's Office-Federal Office for Scientific, Technical and Cultural Affairs," and the European ESF-Vortex Matter. L.R.E.C. was supported by the Brazilian Science Agency CAPES. B.J.B. also acknowledges support from FWO-Vlaanderen. We acknowledge discussions with A. Geim.

\section{APPENDIX: VORTEX CONFIGURATIONS IN THE LONDON THEORY}

In order to study the vortex configurations in a thin disk within the London approach we proceeded along the lines of Ref. 13. In a superconducting medium the Gibbs free energy in the London limit is given by

$$
F=\frac{1}{8 \pi} \int d^{3} r\left[\left(\vec{H}-\vec{H}_{0}\right)^{2}+\lambda^{2}|\vec{j}|^{2}\right] .
$$

In the thin-film limit (thickness $d \ll R$ ) and using the fact that $\vec{H}-\vec{H}_{0}=\vec{\nabla} \times\left(\vec{A}-\vec{A}_{0}\right)$, one may write Eq. (A1) as

$$
F=\frac{1}{8 \pi} \int d^{2} \rho\left(\frac{4 \pi}{c} \vec{J}\right) \cdot\left[\left(\vec{A}-\vec{A}_{0}\right)+\frac{4 \pi \Lambda}{c} \vec{J}\right],
$$

where the integration is now to be performed along the thinfilm plane $z=0$. In this formula $\vec{A}_{0}=\vec{H}_{0} \times \vec{\rho} / 2, \Lambda=\lambda^{2} / d$ is the effective London penetration depth, and $\vec{J}=\int_{0}^{d} d z \vec{j} \approx \vec{j} d$ is the sheet current which is constrained inside the film.

In order to study the vortex configurations in thin disks, and for $\Lambda \gg R$, we may take $\vec{A} \approx \vec{A}_{0}$. This allows us to ne- 
glect demagnetization effects. In the London gauge $(\vec{\nabla} \cdot \vec{A}$ $=0$ ) and in the presence of $N$ vortices the London equation can be written as

$$
\vec{J}=\frac{c}{4 \pi \Lambda}\left(\sum_{i=1}^{N} \vec{\Phi}\left(\left|\vec{\rho}-\vec{\rho}_{i}\right|\right)-\vec{A}\right),
$$

where $\rho_{i}$ is the position of the $i$ th vortex, $\vec{\Phi}(\rho)$ $=\hat{\phi} \Phi_{0} / 2 \pi \rho$, and with $\vec{J}(R) \cdot \hat{\rho}=0$ as the boundary condition. In order to fulfill this condition, one may place vortex images at $\left(R / \rho_{i}\right)^{2} \vec{\rho}_{i}$ away from the center of the disk. ${ }^{13}$ Therefore,

$$
\vec{\Phi}=\sum_{i=1}^{N}\left[\Phi\left(\left|\vec{\rho}-\vec{\rho}_{i}\right|\right)-\Phi\left(\left|\vec{\rho}-\left(R / \rho_{i}\right)^{2} \vec{\rho}_{i}\right|\right)\right]
$$

Also, by substituting Eq. (A3) inside Eq. (A2) (and by using $\vec{A} \approx \vec{A}_{0}$ ), one finds

$$
F=\frac{1}{8 \pi \Lambda} \int d^{2} \rho\left(\vec{\Phi}-\vec{A}_{0}\right)^{2}=\frac{2 \pi \Lambda}{c^{2}} \int d^{2} \rho J^{2} .
$$

It is useful to rewrite the free energy in terms of the function $g(\vec{\rho})$, where $\vec{J}=\vec{\nabla} \times(\hat{z} g), g(R, \phi)=0$, and $g(\vec{\rho})$ can be regarded as a local magnetization in a thin film. ${ }^{23}$ Therefore,

$$
\begin{aligned}
F & =\frac{2 \pi \Lambda}{c^{2}} \int d^{2} \rho g(\vec{\rho}) \hat{z} \cdot \vec{\nabla} \times \vec{J} \\
& =\frac{1}{2 c}\left[\Phi_{0} \sum_{i=1}^{N} g\left(\vec{\rho}_{i}\right)-H_{0} \int d^{2} \rho g(\vec{\rho})\right] .
\end{aligned}
$$

By using Eqs. (A3) and (A4) one can show that

$$
\begin{aligned}
g(\vec{\rho})= & \frac{c}{4 \pi \Lambda}\left[\frac{\Phi_{0}}{2 \pi} \sum_{j=1}^{N} \ln \left(\frac{\left|\vec{\rho}-\left(R / \rho_{j}\right)^{2} \vec{\rho}_{j}\right|}{\left|\vec{\rho}-\vec{\rho}_{j}\right|} \frac{\rho_{j}}{R}\right)\right. \\
& \left.-\frac{H_{0}}{4}\left(R^{2}-\rho^{2}\right)\right] .
\end{aligned}
$$

Substituting this formula in Eq. (A6), and considering $H$ in units of $H_{c 2}$, lengths in units of $\xi$, and energy in units of $\Phi_{0}^{2} / 16 \pi^{2} \Lambda=F_{0}(2 \xi / R)^{2}, F$ is written as

$$
\begin{aligned}
F= & \sum_{i=1}^{N} \sum_{j=1}^{N} \ln \left(\frac{\left|\vec{\rho}_{i}-\left(R / \rho_{j}\right)^{2} \vec{\rho}_{j}\right|}{\left|\vec{\rho}_{i}-\vec{\rho}_{j}\right|} \frac{\rho_{j}}{R}\right)-\frac{1}{2} H_{0} \sum_{i=1}^{N}\left(R^{2}-\rho_{i}^{2}\right) \\
& +\left(\frac{R^{2} H_{0}}{4}\right)^{2} .
\end{aligned}
$$

To simplify this expression the following relation is used:

$$
\int d^{2} \rho \ln \left(\frac{\left|\vec{\rho}-\left(R / \rho_{j}\right)^{2} \vec{\rho}_{j}\right|}{\left|\vec{\rho}-\vec{\rho}_{j}\right|}\right)=\frac{\pi}{2}\left(R^{2}-\rho_{j}^{2}\right)+\pi R^{2} \ln \left(\frac{R}{\rho_{j}}\right) .
$$

To remove the divergence in Eq. (A8) a cutoff procedure is employed, in which for $i=j \rightarrow\left|\vec{\rho}_{i}-\vec{\rho}_{j}\right|=a \quad\left(i=j \rightarrow\left|\vec{\rho}_{i}-\vec{\rho}_{j}\right|\right.$ $=a \xi$ in not normalized units). Therefore

$$
\begin{aligned}
F= & \sum_{i=1}^{N}\left\{\ln \left[1-\left(\frac{\rho_{i}}{R}\right)^{2}\right]-\frac{R^{2} H_{0}}{2}\left[1-\left(\frac{\rho_{i}}{R}\right)^{2}\right]\right. \\
& \left.+\sum_{j=1}^{i-1} \ln \left[\frac{\left(\rho_{i} \rho_{j} / R\right)^{2}-2\left(x_{i} x_{j}+y_{i} y_{j}\right)+R^{2}}{\rho_{i}^{2}-2\left(x_{i} x_{j}+y_{i} y_{j}\right)+\rho_{j}^{2}}\right]\right\} \\
& +N \ln (R / a)+\frac{1}{4}\left(\frac{R^{2} H_{0}}{2}\right)^{2} .
\end{aligned}
$$

In the above expression the first two terms represent the interaction energy of each vortex with the surface and the shielding currents, respectively; the third term represents the pairwise interaction (repulsion) among vortices while the fourth term is the energy of the vortex cores and the external magnetic-field energy interaction is given by the last one. Notice that this energy of the vortex cores arises from the cutoff procedure and is therefore strongly dependent on the cutoff value $a \xi$ (the actual energy associated with vortex cores should be evaluated more accurately by using the Ginzburg-Landau theory). Notice also that if we use $a=1 / 2$ in the cutoff, a contribution $N \ln (2)$ is added to the energy of the system.

In a thin disk, vortices are considered to be distributed in shells. Here, we considered at most two shells, with or without a vortex in the center. For $N_{1}$ vortices in the inner shell and $N_{2}$ in the outer, it is reasonable to have the $j$ th vortex in the outer shell at $\theta_{j}=2 \pi j / N_{2}$ and the $i$ th vortex in the inner shell at $\theta_{i}=2 \pi i / N_{1}+\alpha$. Here, $\alpha$ is the misalignment angle between vortices in the inner and outer shells. A further approximation is to assume perfectly axially symmetric vortex shells. In this case, vortices belonging to the inner shell are at radius $\rho_{1}$ and to the outer at radius $\rho_{2}$, and we can write the Gibbs free energy as

$$
F^{\text {rings }}=F_{1}+F_{2}+F_{12}+F_{\text {center }}+\frac{1}{4}\left(\frac{R^{2} H_{0}}{2}\right)^{2},
$$

where

$$
\begin{aligned}
F_{\gamma}= & N_{\gamma}\left\{\ln \left[1-\left(\frac{\rho_{\gamma}}{R}\right)^{2}\right]-\frac{R^{2} H_{0}}{2}\left[1-\left(\frac{\rho_{\gamma}}{R}\right)^{2}\right]\right. \\
& +\frac{1}{2} \sum_{n=1}^{N_{\gamma}-1} \ln \left|\frac{\left(\rho_{\gamma}^{2} / R\right)^{2}+R^{2}-2 \rho_{\gamma}^{2} \cos \left(2 \pi n / N_{\gamma}\right)}{2 \rho_{\gamma}^{2}\left[1-\cos \left(2 \pi n / N_{\gamma}\right)\right]}\right| \\
& +\ln (R / a)\},
\end{aligned}
$$

for $\gamma=1,2$,

$$
F_{12}=\sum_{i=1}^{N_{1}} \sum_{j=1}^{N_{2}} \ln \left|\frac{\left(\rho_{1} \rho_{2} / R\right)^{2}+R^{2}-2 \rho_{1} \rho_{2} \cos \theta_{i j}}{\rho_{1}^{2}+\rho_{2}^{2}-2 \rho_{1} \rho_{2} \cos \theta_{i j}}\right|,
$$

and

$$
F_{\text {center }}=-\frac{R^{2} H_{0}}{2}-2\left[N_{1} \ln \left(\frac{\rho_{1}}{R}\right)+N_{2} \ln \left(\frac{\rho_{2}}{R}\right)\right]+\ln (R / a) .
$$


Here, $\theta_{i j}=2 \pi\left(i / N_{1}-j / N_{2}\right)+\alpha$ and $F_{\text {center }}=0$ in the absence of a vortex in the center of the disk. The term $F_{\gamma}$ represents the energy from the interaction among vortices within the same ring $\gamma$ and $F_{12}$, the interaction among vortices of different rings. This can be further generalized to $M$ rings by considering the sum of the $M$

*Electronic address: ben.baelus@ua.ac.be

†Electronic address: leonardo.cabral@ua.ac.be; on leave from Departamento de Física, Universidade Federal de Pernambuco, 50670-901, Recife, Brazil.

FElectronic address: francois.peeters@ua.ac.be

${ }^{1}$ R.C. Ashoori, Nature (London) 379, 413 (1996).

${ }^{2}$ V.M. Bedanov and F.M. Peeters, Phys. Rev. B 49, 2667 (1994).

${ }^{3}$ A.K. Geim, I.V. Grigorieva, S.V. Dubonos, J.G.S. Lok, J.C. Maan, A.E. Filippov, and F.M. Peeters, Nature (London) 390, 256 (1997).

${ }^{4}$ P. Singha Deo, V.A. Schweigert, F.M. Peeters, and A.K. Geim, Phys. Rev. Lett. 79, 4653 (1997).

${ }^{5}$ V.A. Schweigert and F.M. Peeters, Phys. Rev. B 57, 13817 (1998).

${ }^{6}$ V.A. Schweigert, F.M. Peeters, and P. Singha Deo, Phys. Rev. Lett. 81, 2783 (1998).

${ }^{7}$ V.A. Schweigert and F.M. Peeters, Phys. Rev. Lett. 83, 2409 (1999).

${ }^{8}$ A.K. Geim, S.V. Dubonos, I.V. Grigorieva, K.S. Novoselov, F.M. Peeters, and V.A. Schweigert, Nature (London) 407, 55 (2000).

${ }^{9}$ J.J. Palacios, Phys. Rev. Lett. 84, 1796 (2000).

${ }^{10}$ P.G. de Gennes, Superconducting of Metals and Alloys (Ben- terms coming from interactions within the ring and the $M ! / 2 !(M-2)$ ! terms from the interactions between two rings. Also it should be pointed out that the above formula reduces to the one studied by Buzdin and Brison ${ }^{13}$ when only one ring and a possible vortex in the center of the disk are considered.

${ }^{11}$ M. Tinkham, Introduction to Superconductivity (McGraw-Hill, New York, 1975).

${ }^{12}$ A. A. Abrikosov, Fundamentals of the Theory of Metals (NorthHolland, Amsterdam, 1986).

${ }^{13}$ A.I. Buzdin and J.P. Brison, Phys. Lett. A 196, 267 (1994).

${ }^{14}$ J.J. Palacios, Phys. Rev. B 58, R5948 (1998).

${ }^{15}$ B.J. Baelus, F.M. Peeters, and V.A. Schweigert, Phys. Rev. B 63, 144517 (2001).

${ }^{16}$ A.K. Geim, S.V. Dubonos, J.J. Palacios, I.V. Grigorieva, M. Henini, and J.J. Schermer, Phys. Rev. Lett. 85, 1528 (2000).

${ }^{17}$ V.A. Schweigert and F.M. Peeters, Physica C 332, 426 (2000).

${ }^{18}$ R. Kato, Y. Enomoto, and S. Maekawa, Phys. Rev. B 47, 8016 (1993).

${ }^{19}$ B.J. Baelus and F.M. Peeters, Phys. Rev. B 65, 104515 (2002).

${ }^{20}$ Minghui Kong, B. Partoens, and F.M. Peeters, Phys. Rev. E 65, 046602 (2002).

${ }^{21}$ B.J. Baelus, S.V. Yampolskii, F.M. Peeters, E. Montevecchi, and J.O. Indekeu, Phys. Rev. B 65, 024510 (2002).

${ }^{22}$ P.A. Venegas and E. Sardella, Phys. Rev. B 58, 5789 (1998).

${ }^{23}$ E.H. Brandt, Phys. Rev. Lett. 74, 3025 (1995). 